\title{
Tracking an electronic wave packet in the vicinity of a conical intersection
}

Da-Long Qi, Hong-Guang Duan, Zhen-Rong Sun, R. J. Dwayne Miller, and Michael Thorwart

Citation: The Journal of Chemical Physics 147, 074101 (2017); doi: 10.1063/1.4989462

View online: https://doi.org/10.1063/1.4989462

View Table of Contents: http://aip.scitation.org/toc/jcp/147/7

Published by the American Institute of Physics

\section{Articles you may be interested in}

Coupled electron-nuclear quantum dynamics through and around a conical intersection

The Journal of Chemical Physics 147, 064302 (2017); 10.1063/1.4989780

Electron-nuclear wave-packet dynamics through a conical intersection

The Journal of Chemical Physics 146, 074304 (2017); 10.1063/1.4975811

Beyond Born-Oppenheimer theory for ab initio constructed diabatic potential energy surfaces of singlet $\mathrm{H}_{3}^{+}$to study reaction dynamics using coupled 3D time-dependent wave-packet approach

The Journal of Chemical Physics 147, 074105 (2017); 10.1063/1.4998406

Dynamic mapping of conical intersection seams: A general method for incorporating the geometric phase in adiabatic dynamics in polyatomic systems

The Journal of Chemical Physics 147, 044109 (2017); 10.1063/1.4990002

Signatures of vibronic coupling in two-dimensional electronic-vibrational and vibrational-electronic spectroscopies

The Journal of Chemical Physics 147, 094202 (2017); 10.1063/1.4991745

On the adiabatic representation of Meyer-Miller electronic-nuclear dynamics

The Journal of Chemical Physics 147, 064112 (2017); 10.1063/1.4995301

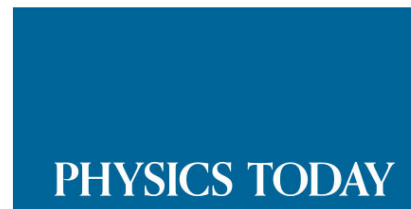

ADVANCED LIGHT CURE ADHESIVES

Take a closer look at what these environmentally friendly adhesive systems can do
READ NOW

PRESENTED BY

Q MASTERBOND 


\title{
Tracking an electronic wave packet in the vicinity of a conical intersection
}

\author{
Da-Long Qi, ${ }^{1,2, a)}$ Hong-Guang Duan, ${ }^{2,3,4, a)}$ Zhen-Rong Sun, ${ }^{1}$ R. J. Dwayne Miller, ${ }^{2,4,5}$ \\ and Michael Thorwart ${ }^{3,4}$ \\ ${ }^{1}$ State Key Laboratory of Precision Spectroscopy, School of Physics and Material Science, East China Normal \\ University, 3663 North Zhongshan Road, 200062 Shanghai, China \\ ${ }^{2}$ Max Planck Institute for the Structure and Dynamics of Matter, Luruper Chaussee 149, 22761 Hamburg, \\ Germany \\ ${ }^{3}$ I. Institut für Theoretische Physik, Universität Hamburg, Jungiusstraße 9, 20355 Hamburg, Germany \\ ${ }^{4}$ The Hamburg Center for Ultrafast Imaging, Luruper Chaussee 149, 22761 Hamburg, Germany \\ ${ }^{5}$ The Departments of Chemistry and Physics, University of Toronto, 80 St. George Street, Toronto, \\ Ontario M5S 3H6, Canada
}

(Received 9 June 2017; accepted 1 August 2017; published online 15 August 2017)

\begin{abstract}
This work treats the impact of vibrational coherence on the quantum efficiency of a dissipative electronic wave packet in the vicinity of a conical intersection by monitoring the time-dependent wave packet projection onto the tuning and the coupling mode. The vibrational coherence of the wave packet is tuned by varying the strength of the dissipative vibrational coupling of the tuning and the coupling modes to their thermal baths. We observe that the most coherent wave packet yields a quantum efficiency of $93 \%$, but with a large transfer time constant. The quantum yield is dramatically decreased to $50 \%$ for a strongly damped incoherent wave packet, but the associated transfer time of the strongly localized wave packet is short. In addition, we find for the strongly damped wave packet that the transfer occurs via tunneling of the wave packet between the potential energy surfaces before the seam of the conical intersection is reached and a direct passage takes over. Our results provide direct evidence that vibrational coherence of the electronic wave packet is a decisive factor which determines the dynamical behavior of a wave packet in the vicinity of the conical intersection. Published by AIP Publishing. [http://dx.doi.org/10.1063/1.4989462]
\end{abstract}

\section{INTRODUCTION}

A conical intersection $(\mathrm{CI})$ is a degenerate point of two potential energy surfaces (PESs) in the configuration space of a polyatomic molecule. This degeneracy between two PESs induces a strong nonadiabatic coupling between the electronic states, such that the Born-Oppenheimer approximation breaks down and, in general, an ultrafast electronic and vibrational relaxation results. ${ }^{1-4}$ Due to the strong nonadiabatic coupling of the electronic to the vibrational degrees of freedom, the molecular electronic dynamics can be monitored by studying the vibrational coherence of the electronic wave packet along the reaction coordinates.

Various coherent spectroscopic techniques are useful tools to study the wave packet dynamics in the vicinity of the CI. ${ }^{5}$ Among them, the femtosecond stimulated Raman spectroscopy (FSRS) is a powerful tool to reveal the changes of the vibrational coherence in the molecule moving in the vicinity of the CI. ${ }^{6,7}$ FSRS has been used to study the photoisomerization of rhodopsin, which was estimated to occur within a time scale of $200 \mathrm{fs}$ or less. ${ }^{8}$ The structural evolution of the stilbene photoisomerization has been tracked by the gradual shift of the associated vibrational frequency over time. ${ }^{9}$ The proton transfer in the green fluorescent protein ${ }^{10}$ and the repair mechanism of thymine dimer ${ }^{11}$ have

a) D.-L. Qi and H.-G. Duan contributed equally to this work. been revealed by the FSRS. More recently, two-dimensional time-resolved stimulated Raman spectroscopy has been applied to measure the vibrational coherence of a chargetransfer dimer. Thereby, the anharmonicity of the vibrational coherence on the excited PESs has been clearly revealed. ${ }^{12}$ Moreover, the transient absorption spectroscopy is another diagnostic tool to uncover how the atom in the reactants moves during the formation of the product. The motion of the electronic wave packet in the primary photoisomerization reaction has been studied by the transient absorption measurement. ${ }^{13} \mathrm{~A}$ frequency shift due to the stimulated emission of the electronic wave packet on the excited state surface was observed. Furthermore, by tracking the vibrational coherence on the PESs, it was shown that the stretch mode in the retinal molecule is largely unaffected by the presence of the CI, while the hydrogen-out-of-plane (HOOP) mode is strongly damped after passing through the CI. ${ }^{14}$ In addition, the high-order anharmonic couplings between different vibrational modes on the excited state surface have been partially resolved by the transient absorption spectroscopy. ${ }^{15}$ Recently, using an ultrashort pulse and exploiting the sensitivity of the heterodyne detected transient grating approach, the local vibrational coherence of the effective modes has been identified to drive the primary photoisomerization in vertebrate vision. ${ }^{16}$ This measurement has refined the time scale of the primary step in vision, and the passage through the CI was shown to occur within $50 \mathrm{fs}$. This has also been confirmed by the two-dimensional electronic spectroscopy, ${ }^{17}$ which indicates that the relevant 
reaction dynamics occurs under the vibrational coherent process before decoherence of the reaction coordinates sets in. This directly implies that the vibrational coherence can be used to control the dynamics and the efficiency of the photoisomerization. The coherent control of the primary step of the photoisomerization of rhodopsin has been demonstrated by modulating the phase and the amplitude of the excitation laser pulse in the transient absorption spectroscopy. ${ }^{18}$ The isomerization efficiency has been increased and decreased by $20 \%$ by using the optimal or anti-optimal pulse shape. The dominant contribution was from amplitude shaping in which the shaped pulse matched the period of the key tuning mode of the reaction coordinate, arguing for the importance of vibrational resonances in passage through the $\mathrm{CI}$. The phase aspect of the degree of control was only on the order of $5 \%$. It was not possible to observe the same effects with all phase control. ${ }^{19}$ Although the phase-only aspect was only a few percent, it could be argued that the noise level of $4 \%$ in this experiment would make it difficult to observe such an effect. The question then is what is the physics at work and to what degree can the reaction dynamics be controlled at CIs.

Theoretically, in a minimal model, the physics of a CI can be described by a two-state two-mode model. ${ }^{20}$ The transient absorption spectroscopy of the two-state two-mode model has been calculated for the cis-trans isomerization. ${ }^{21}$ The wave packet on the PESs is projected onto the effective reaction coordinates of the tuning and coupling modes, respectively. Its dynamics was calculated by using quantum master equations, e.g., by the Redfield master equation ${ }^{22}$ or the hierarchy equation of motion. ${ }^{23}$ This model has been further simplified by transferring the tuning and coupling modes to the harmonic bath and solving the resulting non-Markovian dynamics by a numerically exact approach. ${ }^{24}$ By this, the model, in principle, can describe the configuration with multiple CIs between the PESs, which is more close to a realistic situation. Moreover, 2D electronic spectroscopy has been applied to study the electronic wave packet dynamics in the vicinity of the CI. ${ }^{25-27}$ It has been found that the excited-state absorption becomes significant when compared to the model where only vibrational coupling is present. In addition, it has been proposed to use vibrational coherence in $2 \mathrm{D}$ spectra to measure the interstate coupling near a CI. ${ }^{28}$ Also attosecond spectroscopy has been proposed to use to detect the CI by monitoring the electronic coherence at the degenerate point between the adjacent PESs. ${ }^{29}$ More recently, a numerical calculation has shown that photorelaxation in uracil can be fully modulated to form the long-lived excited state by a properly shaped laser pulse. ${ }^{30}$

The kinetic energy of the wave packet on the PESs is considered as one tool to control the photoisomerization efficiency in the CI. ${ }^{31}$ More details of the coherent control of the electron-nuclear dynamics at the CI have been provided recently. ${ }^{32}$ In Ref. 33, some of us have investigated the role of vibrational coherence of an electronic wave packet moving in the vicinity of a $\mathrm{CI}$ and have provided evidence that the quantum yield and the isomerization rate both are strongly related to the vibrational coherence. This was shown by calculating the transient absorption and the $2 \mathrm{D}$ electronic spectra of the three-state two-mode model. The time evolution of the electronic wave packet in the transient absorption spectrum has been tracked and, counterintuitively, it was found that the more coherent wave packet results in a higher quantum yield, but with a larger isomerization time constant. However, the full picture of the nonadiabatic dynamics of an electronic wave packet near a CI is still unclear and the direct evidence of the impact of vibrational coherence on the quantum efficiency is still missing.

In this paper, we study the impact of vibrational coherence on the quantum yield and the isomerization rate of an electronic wave packet moving in the vicinity of a CI by monitoring the nonadiabatic dynamics of the wave packet projected onto the tuning and the coupling mode. This allows us to directly visualize the quantum yield and its isomerization time constant (or, rate). By varying the strength of the dissipative coupling of the vibrational modes to their respective baths, we find that the quantum yield can be significantly enhanced by an increased vibrational coherence of the wave packet. In fact, the generated quantum yield can reach up to $93 \%$ for the most coherent wave packet, and it can be significantly reduced to $50 \%$ for an electronic wave packet which experiences strong vibrational damping. In addition, we find in the case of stronger damping that the electronic wave packet directly penetrates the higher-lying PES to the lower electronic state via tunneling, before the remaining parts of the wave packet reach the seam of the CI and can pass directly through. The direct tracking of the dissipative wave packet motion provides an elegant experimental tool to reveal the role of vibrational dissipation at a CI. This strong correlation of the coherence of the key modes involved in the passage through the CI is effectively related to steering the system away from coupling to vibrational modes not involved in passage through the CI. This finding recognizes that the dominant loss of coherence in vibrational coordinate space is through anharmonic coupling to the vibrational phase space orthogonal to reactive trajectories.

\section{MODEL}

We start with the two-state two-mode model in which a spectroscopically accessible CI is assumed with two electronic states $|e 1\rangle$ and $|e 2\rangle$. Moreover, we assume the molecule to be coupled to a dissipative bath, which is composed of infinitely many harmonic oscillators. Thus, the total Hamiltonian can be written as $H=H_{\text {mol }}+H_{\text {env }}$. The molecular Hamiltonian is given by $H_{\mathrm{mol}}=H_{\mathrm{e} 1}+H_{\mathrm{e} 2}$ with $H_{\mathrm{e} 1}=|e 1\rangle\left(h_{1}-\epsilon / 2\right)\langle\mathrm{e} 1|$ and $H_{\mathrm{e} 2}=|e 2\rangle\left(h_{2}+\epsilon / 2\right)\langle e 2|+(|e 1\rangle V\langle e 2|+$ h.c. $)$. Here, $\epsilon$ is the energy gap between two electronic states, $|e 1\rangle$ and $|e 2\rangle$. The terms $h_{1}$ and $h_{2}$ are the vibrational Hamiltonians associated to the electronic states $|e 1\rangle$ and $|e 2\rangle$, respectively. They represent two vibrational modes approximated as harmonic, the tuning mode $Q_{t}$ and the coupling mode $Q_{c}$, respectively. They can be written as $h_{1}=\frac{1}{2} \sum_{i=t, c} \Omega_{i}\left(P_{i}^{2}+Q_{i}^{2}\right)-\kappa Q_{t}$ and $h_{2}=\frac{1}{2} \sum_{i=t, c} \Omega_{i}\left(P_{i}^{2}+Q_{i}^{2}\right)+\kappa Q_{t}$. Herein, $\kappa$ is the strength of the vibronic coupling of the two PESs. The electronic coupling between two PESs is assumed to linearly depend on $Q_{c}$, such as $V=\Lambda Q_{c}$ with the electronic coupling strength $\Lambda$. We assume that the tuning and the coupling mode each couples 
to its own bath consisting of an infinite number of harmonic oscillators which are linearly coupled to the given mode, with the overall Hamiltonian,

$$
\begin{aligned}
H_{\mathrm{env}}= & \sum_{\alpha}\left[\frac{p_{\alpha}^{2}}{2 m_{\alpha}}+\frac{m_{\alpha} \omega_{\alpha}^{2}}{2}\left(x_{\alpha}+\frac{c_{\alpha} Q_{c}}{m_{\alpha} \omega_{\alpha}^{2}}\right)^{2}\right. \\
& \left.+\frac{q_{\alpha}^{2}}{2 M_{\alpha}}+\frac{M_{\alpha} v_{\alpha}^{2}}{2}\left(y_{\alpha}+\frac{d_{\alpha} Q_{t}}{M_{\alpha} v_{\alpha}^{2}}\right)^{2}\right]
\end{aligned}
$$

Here, the momenta of the bath oscillators are denoted as $p_{\alpha}$ and $q_{\alpha}$, while their coordinates, masses, and frequencies are denoted by $x_{\alpha}, m_{\alpha}, \omega_{\alpha}$ and $y_{\alpha}, M_{\alpha}, v_{\alpha}$, respectively. The respective coupling constants are $c_{\alpha}$ and $d_{\alpha}$. The two parts of the bath are characterized by the spectral densities $J_{c}(\omega)$ $=\frac{1}{2} \sum_{\alpha} \frac{c_{\alpha}^{2}}{m_{\alpha} \omega_{\alpha}} \delta\left(\omega-\omega_{\alpha}\right)$ and $J_{t}(\omega)=\frac{1}{2} \sum_{\alpha} \frac{d_{\alpha}^{2}}{M_{\alpha} v_{\alpha}} \delta\left(\omega-v_{\alpha}\right)$. Throughout this work, we assume that both the tuning and coupling mode experience fluctuations with an Ohmic spectral density $J_{t / c}(\omega)=\eta_{t / c} \omega \exp \left(-\omega / \omega_{c}\right)$. The $\eta_{t / c}$ are the damping strengths for the tuning and coupling modes, respectively, $\omega_{c}$ is the cutoff frequency which is assumed, for simplicity, as equal for the two baths. The reorganization energies are given by $R_{t / c}=2 \eta_{t / c} \hbar \omega_{c} / \pi$.

In this study, we assume the initial wave packet to be prepared in the state $|e 2,0\rangle$, such that the density matrix at initial time $t=0$ can be written as $\rho(0)=\left|e 2,0_{c}, 0_{t}\right\rangle\left\langle e 2,0_{c}, 0_{t}\right|$. Here, $0_{c}$ and $0_{t}$ are the vibrational ground states of the coupling and tuning modes, respectively. In order to obtain the time-dependent position of the electronic wave packet on the PES, we need to project the wave packet on the corresponding coordinates in the adiabatic basis. The projection yields the two reduced probability densities,

$$
\begin{aligned}
& P_{k}^{a d}\left(Q_{c}, t\right)=\int d Q_{t}\left\langle Q_{c}\left|\left\langle Q_{t}\left|\left\langle\tilde{e}_{k}|\rho(t)| \tilde{e}_{k}\right\rangle\right| Q_{t}\right\rangle\right| Q_{c}\right\rangle, \\
& P_{k}^{a d}\left(Q_{t}, t\right)=\int d Q_{c}\left\langle Q_{c}\left|\left\langle Q_{t}\left|\left\langle\tilde{e}_{k}|\rho(t)| \tilde{e}_{k}\right\rangle\right| Q_{t}\right\rangle\right| Q_{c}\right\rangle,
\end{aligned}
$$

of the coupling mode and the tuning mode, respectively. Here, $\left|\tilde{e}_{k=1,2}\right\rangle$ denotes the electronic wave function in the adiabatic basis. The transformation of the wave function from the diabatic to the adiabatic basis is given by $\left|\tilde{e}_{k}\right\rangle$ $=\sum_{k^{\prime}=e 1, e 2} S\left(Q_{c}, Q_{t}\right)_{k k^{\prime}}\left|e_{k^{\prime}}\right\rangle$, and the transfer matrix $S\left(Q_{c}, Q_{t}\right)$ can be found in Ref. 34. We assume a vibronic coupling strength of $\kappa=1000 \mathrm{~cm}^{-1}$ of the tuning mode $Q_{t}$ and a vibronic coupling strength of $\Lambda=200 \mathrm{~cm}^{-1}$ of the coupling mode, $\Omega_{t}=500 \mathrm{~cm}^{-1}$ and $\Omega_{c}=900 \mathrm{~cm}^{-1}$, which are typical frequencies of the molecular vibrations. ${ }^{12,15,35}$ The energy gap between the two PESs is set to $\epsilon=2000 \mathrm{~cm}^{-1}$. In this work, all the calculations have been carried out at room temperature $(T=300 \mathrm{~K})$. In order to realize a sufficiently large eigenbasis of the molecular Hamiltonian for converged results, 30 and 6 vibrational levels are included for the tuning and coupling modes, respectively. The total molecular Hamiltonian matrix is 360 dimensional. We use the time non-local (TNL) quantum master equation $^{36,37}$ for the calculation of the full dynamics of the electronic wave packet. Based on the above parameters, we plot the PES-configuration of the two electronic states in Fig. 1. By this, the quantum yield can be measured by determining the ratio of the magnitudes of the wave packet at $A$ and the total magnitude $\mathrm{A}+\mathrm{B}$, as sketched in Fig. 1(a). This yields
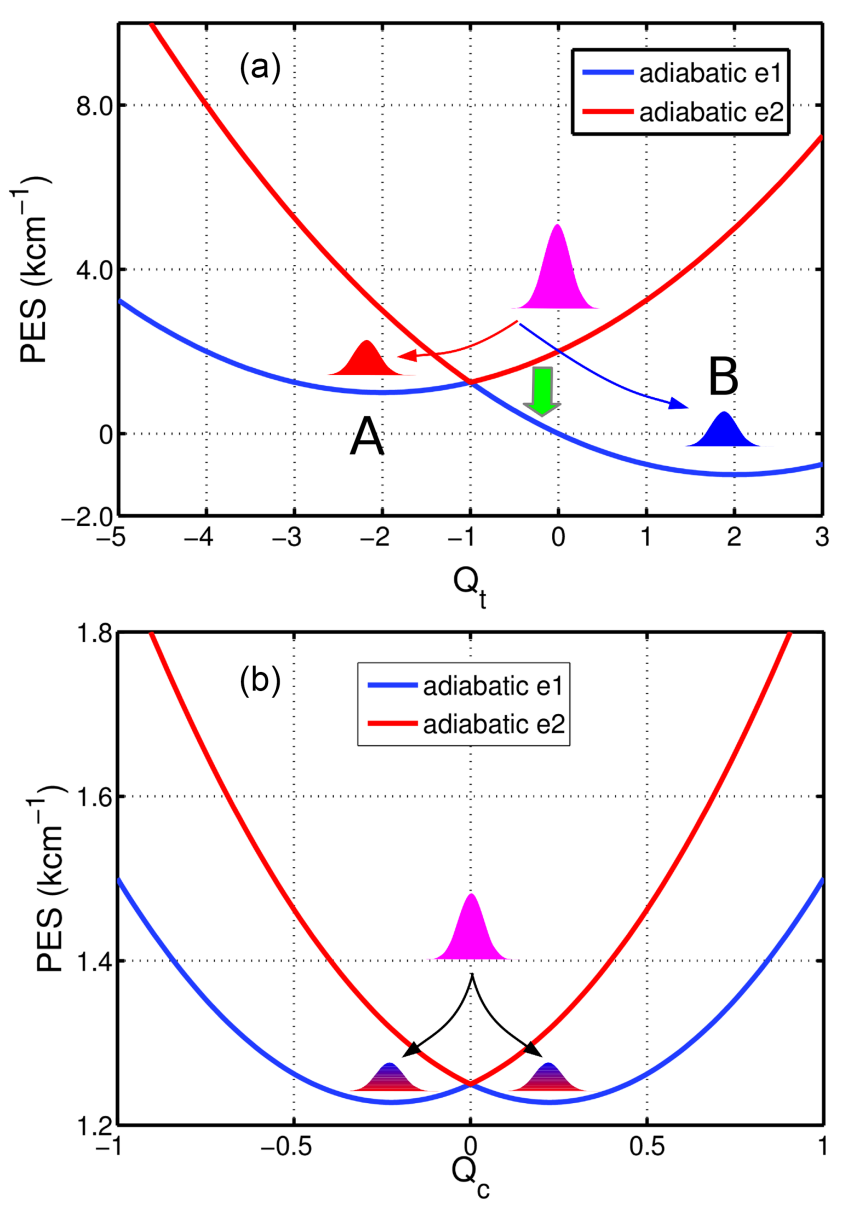

FIG. 1. Configuration of the potential energy surfaces and the conical intersection along the tuning (a) and the coupling (b) modes. The conical intersection is located at the tuning coordinate $Q_{t}=-1$ and at the center of the coupling mode at $Q_{c}=0$. The quantum yield is calculated by the magnitudes of the wave packets at $A$ and $B$ of the state $|\tilde{e} 1\rangle$ according to $Y=\frac{A}{A+B}$.

$Y=\frac{A}{A+B}$, which, for instance, can be used to quantify the efficiency of photoisomerization from the initial to the product state.

\section{WEAK VIBRATIONAL DAMPING}

On the basis of this model, we study the impact of vibrational coherence of the wave packet on the kinetics around the CI. For this, we vary the vibrational damping constant $\eta_{t / c}$. We begin with the weak damping case and choose $\eta_{t}=\eta_{c}=0.5$ and $\omega_{c}=50 \mathrm{~cm}^{-1}$. We calculate the dynamics of the wave packet and project it to the tuning and coupling coordinates. The results of the two reduced probability densities are shown in Fig. 2 in the color scale. In Fig. 2(a), the initial wave packet prepared in the state $|\tilde{e} 2\rangle$ was initially localized at $Q_{t}=0$. It starts to move to the left side and passes through the CI [see Fig. 1(a), position at $\left.Q_{t}=-1\right]$ within the first 20 fs. Consequently, the wave packet of the state $|\tilde{e} \overline{2}\rangle$ completely disappears on the upper PES, while, at the same time, the wave packet starts to grow at $Q_{t}=-1$ on the $|\tilde{e} 1\rangle$-PES. With growing time, it continues to move further to the left side until it reaches its maximal displacement at $Q_{t}=-6$. Then, it turns around, moves towards the center again, and penetrates the PES to come back to the upper electronic PES of $|\tilde{e} 2\rangle$. This can be observed in 

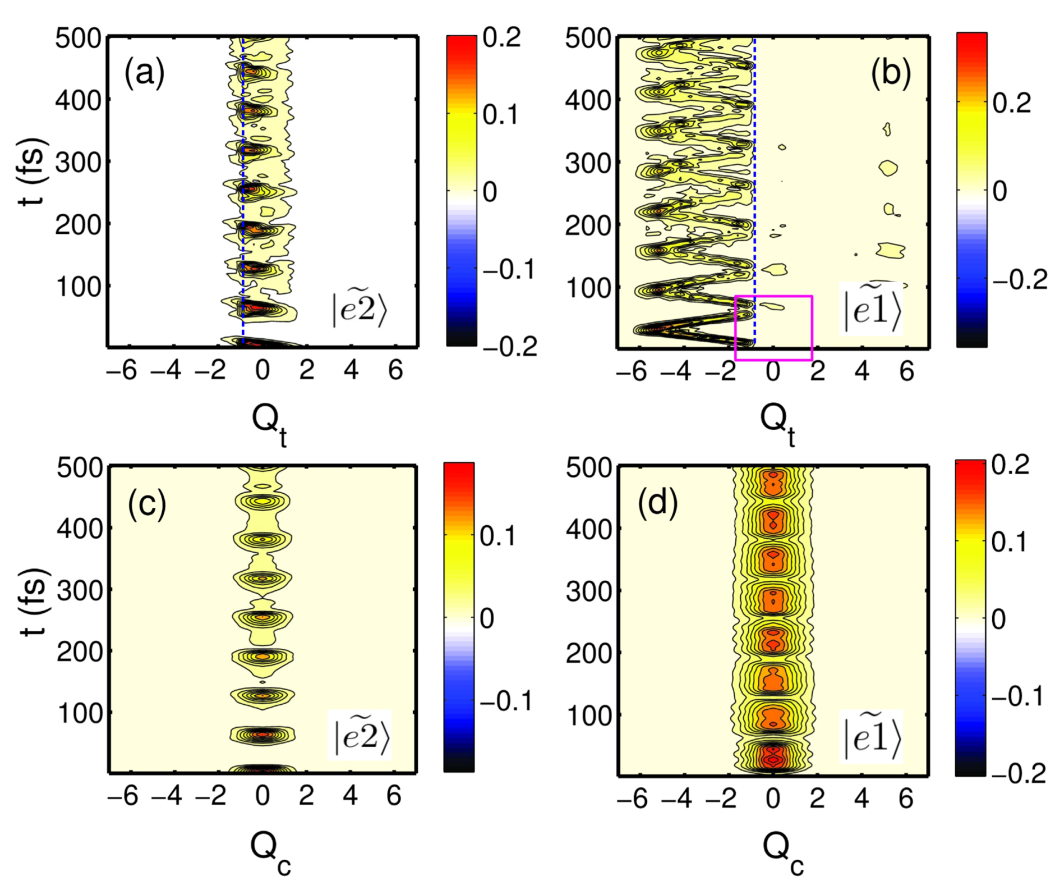

FIG. 2. Time evolution of the probability densities of the underdamped electronic wave packet on the $|\tilde{e} 2\rangle$-PES and the $|\tilde{e} 1\rangle$-PES along the tuning mode $Q_{t}$ and the coupling mode $Q_{c}$ : (a) $P_{2}^{a d}\left(Q_{t}, t\right)$, (b) $P_{1}^{a d}\left(Q_{t}, t\right)$, (c) $P_{2}^{a d}\left(Q_{c}, t\right)$, and (d) $P_{1}^{a d}\left(Q_{c}, t\right)$. The parameters are $\eta_{t}=\eta_{c}=0.5$ and $\omega_{c}=50 \mathrm{~cm}^{-1}$ in the Ohmic spectral density.
Fig. 2(a) to occur at $\sim 60$ fs. After that, the wave packet moves towards the $\mathrm{CI}$ again and repeats the kinetics as described until the end of the considered time window of $500 \mathrm{fs}$. During this motion, vibrational coherence can be clearly identified and shows a period of $\sim 65 \mathrm{fs}$ in the wave-packet dynamics on the $|\tilde{e} 1\rangle$-PES. This value coincides with the vibrational frequency of the tuning mode being set to $500 \mathrm{~cm}^{-1}$. Moreover, the transfer time $\tau$ and the quantum yield $Y$ of the wave packet between the two PESs can be clearly resolved. ${ }^{38}$ First, the quantum yield is quite high in this case of weak damping since the electronic wave packet is mostly located in the left region of the $|\tilde{e} 1\rangle$ PES in Fig. 2(b). Moreover, an effective potential barrier can be identified at $Q_{t}=-1$ which blocks the wave packet motion further to the right side. The quantum yield can be detected by integrating over one oscillation period of the wave packet at the left side up to $Q_{t}=-1$ around the waiting time of $500 \mathrm{fs}$. The associated transfer time can be easily determined by the exponential fitting of the kinetics of the integrated wave packet at the left side. In the case of weak damping, we find $Y=93 \%$ and a transfer time $\tau=920 \mathrm{fs}$.

The wave packet also shows interesting dynamics along the coupling mode direction, see Figs. 2(c) and 2(d). At the beginning, the wave packet is localized at the center $Q_{c}=0$. It penetrates the CI and leaves the PES of $|\tilde{e}\rangle$ within $20 \mathrm{fs}$. At the same time, the wave packet appears on the $|\tilde{e} 1\rangle$-PES and reaches the maximum probability at $25 \mathrm{fs}$. Then, the wave packet starts to decay from the $|\tilde{e} 1\rangle$-PES and moves back to the $|\tilde{e} 2\rangle$-surface. Also these kinetics are repeated. The period of this oscillation is $65 \mathrm{fs}$ which coincides with the period of the tuning mode. Interestingly, a coherent vibrational oscillation along the coupling mode direction cannot be identified since the wave packet splits into two parts and shows different frequencies of the oscillation on the $|\tilde{e} 1\rangle$ PES in the adiabatic basis [see Fig. 1(b)]. This is consistent with the theoretical and experimental results of Refs. 14, 22, and 33 .

\section{STRONG VIBRATIONAL DAMPING}

For the case of strong vibrational damping, we choose the parameters $\eta_{t}=\eta_{c}=2$ and $\omega_{c}=200 \mathrm{~cm}^{-1}$. The results for the wave-packet dynamics are shown in Fig. 3. In Fig. 3(a), the wave packet population on the $|\tilde{e} 2\rangle$-surface shows a much stronger damped dynamics. The wave packet only shows three clear oscillation periods up to $\sim 120 \mathrm{fs}$ and then disintegrates and disappears after $200 \mathrm{fs}$. This shows that the wave packet has completely passed through the CI to the PES of $|\tilde{e} 1\rangle$. In Fig. 3(b), the wave packet dynamics is similar to the one on the $|\tilde{e} 2\rangle$-PES and also shows three periods of vibrational coherence and gets completely disintegrated afterwards. Yet, it can be clearly observed that the transfer time is significantly shorter, but with a smaller efficiency in view of the quantum yield as compared to the weak damping case in Fig. 2. Similarly, for the wave packet dynamics along the coupling mode, we also observe that the transfer happens faster than before.

In Fig. 3(d), we see that the wave packet on the $|\tilde{e} 1\rangle$ surface is split into two parts at times $>400$ fs. This is a clear signature of the geometric (or Berry) phase. ${ }^{39-42}$ When the wave packet travels on the two opposite sides around the CI, it can interfere destructively at $Q_{c}=0$ due to the phase difference of $\pi$ acquired along the two different pathways. Thus, the probability density gets reduced at $Q_{c}=0$, and the wave packet develops two maxima symmetrically located at $Q_{c} \neq 0$.

Clearly, the geometric phase does not occur in the weak damping case [Fig. 2(d)] since the electronic wave packet is almost completely localized on the left side of the $|\tilde{e} 1\rangle$-PES. In order to quantify this, we fit the kinetics of the electronic wave packet on the $|\tilde{e} 1\rangle$-surface to an exponential function and obtain the transfer time constant $\tau$. The kinetics extracted from the left and the right side of the $\mathrm{CI}$ (which are separated by the barrier at $Q_{t}=-1$ ) show comparable magnitudes. Furthermore, 

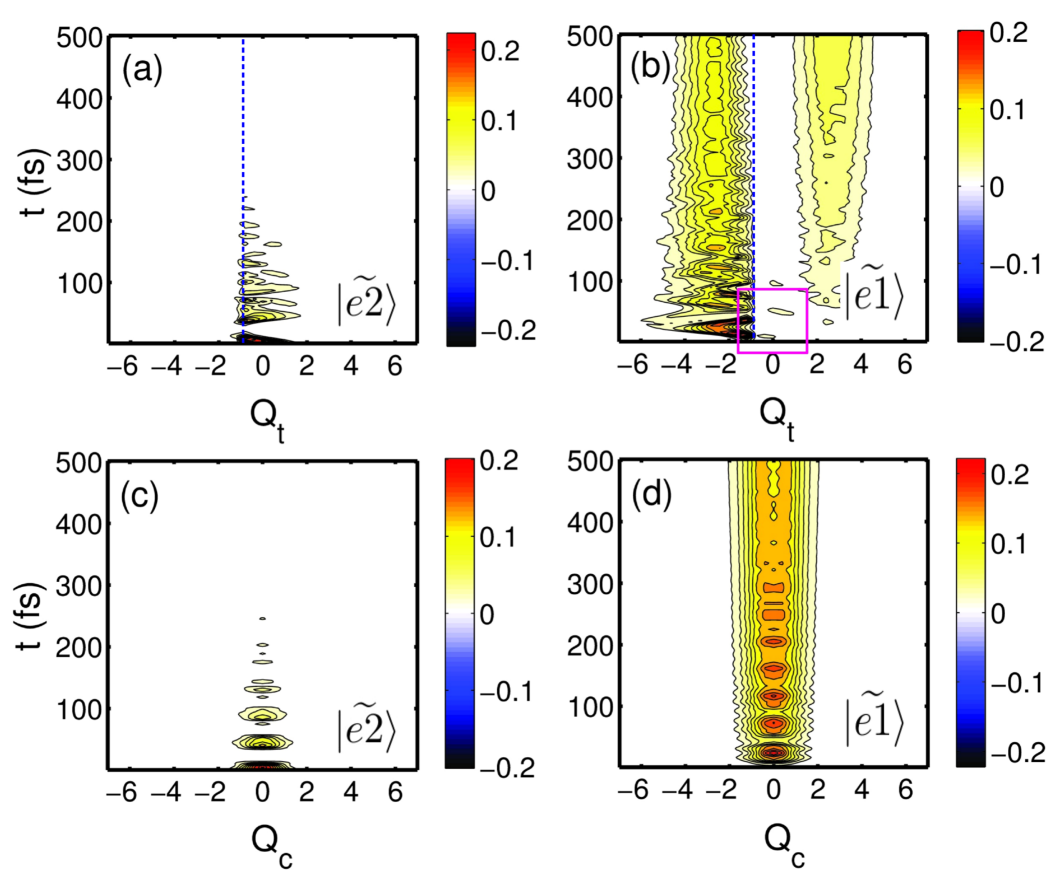

FIG. 3. Same as Fig. 2, but for stronger vibrational damping. The parameters are $\eta_{t}=\eta_{c}=2$ and $\omega_{c}=200$ $\mathrm{cm}^{-1}$. we determine the quantum yield $Y$ by integrating over the probability density (separated by the barrier line at $Q_{t}=-1$ ) at time $t=500 \mathrm{fs}$. The quantum yield decreases to $Y=50.4 \%$ in the case of strong damping as compared to $Y=93 \%$ in the weak damping case.

For a complete picture, we calculate the electronic wave packet dynamics for a wide range of damping parameters, $\eta_{t / c}$ and $\omega_{c}$. The quantum yields and the associated transfer times are listed in Table I. We find that both the transfer time and the quantum yield almost monotonously decrease with increasing damping. Thus, a less damped and hence a more coherent wave packet results in a higher quantum yield but with slower transfer process. This is in agreement with previous work, ${ }^{33}$ but we now prove the effect on the quantum yield from the viewpoint by explicitly tracking the wave packet motion in position space. Further, we illustrate that the spectroscopic approach is capable of revealing the dynamics in the vicinity of a CI, notwithstanding the experimental issue with rapidly changing spectra.

In addition, the significant difference of the wave-packet dynamics on the $|\tilde{e} 1\rangle$-surface at short times should be noticed between the weak (Fig. 2) and the strong damping cases

TABLE I. The reorganization energy $R_{t / c}$, quantum yield $Y$, and transfer time $\tau$ for the different cases of the damping parameters. We set $\eta_{t}=\eta_{c}$, such that $R_{t}=R_{C}$.

\begin{tabular}{lcccc}
\hline \hline$\eta_{t / c}$ & $\omega_{c}\left(\mathrm{~cm}^{-1}\right)$ & $R_{t / c}\left(\mathrm{~cm}^{-1}\right)$ & $\mathrm{Y}(\%)$ & $\tau(\mathrm{fs})$ \\
\hline 0.5 & 50 & 16 & 93 & 920 \\
0.8 & 80 & 41 & 87 & 600 \\
1.1 & 110 & 77 & 82 & 430 \\
1.4 & 140 & 125 & 77 & 350 \\
1.7 & 170 & 184 & 73 & 300 \\
2.0 & 200 & 255 & 50 & 200 \\
\hline \hline
\end{tabular}

(Fig. 3). We highlight this by the magenta boxes. In the strong damping case, a small probability density of the wave packet appears at $Q_{t}=0$ on the $|\tilde{e} 1\rangle$-surface. However, the wave packet on the upper $|\tilde{e}\rangle$-surface does not reach the seam within that time. This indicates that in the strong damping case, the electronic wave packet can penetrate the PES by tunneling when the two PESs are close together (the region is marked by the green arrow in Fig. 1). In contrast, there is no evidence of this tunneling process in the magenta box of the weak damping case in Fig. 2. To confirm this observation, we increase the electronic coupling between the two PESs to $\Lambda=800 \mathrm{~cm}^{-1}$ and calculate the wave packet dynamics up to $t=500 \mathrm{fs}$. We compare the time evolution of the probability density $P_{1}^{a d}\left(Q_{t}, t\right)$ of the wave packet on the $|\tilde{e} 1\rangle$-surface for the cases $\Lambda=800 \mathrm{~cm}^{-1}$ and $\Lambda=200 \mathrm{~cm}^{-1}$ in Fig. 4. In Fig. 4(a), we can clearly identify the tunneling of the wave packet at the coordinate $Q_{t}=0$. Moreover, it also shows that the wave packet moves to the right side and to the minimum of the $|\tilde{e} 1\rangle$-surface. In contrast, we observe in Fig. 4(b) a much smaller magnitude of the wave packet at $Q_{t}=0$ and no clear evidence of the movement towards the minimum at the position $Q_{t}=2$. Based on these calculations, we confirm that the less coherent wave packet does not show a net passage through the CI. Instead, it tunnels through the PESs when they are close together. In contrast, the more coherent wave packet shows an almost complete passage through the CI. We believe that this is the significant factor which ultimately determines the quantum efficiency of the isomerization and would provide a means for evolutionary optimization of the reaction coordinate. The increase in coherence lifetime of the key reaction modes involved in CI passage in turn necessitates reduced anharmonic coupling to the vibrational density of state orthogonal to the reaction coordinate or use of external field to resonantly drive these modes and decouple from peripheral nonresonant terms. 
(a)

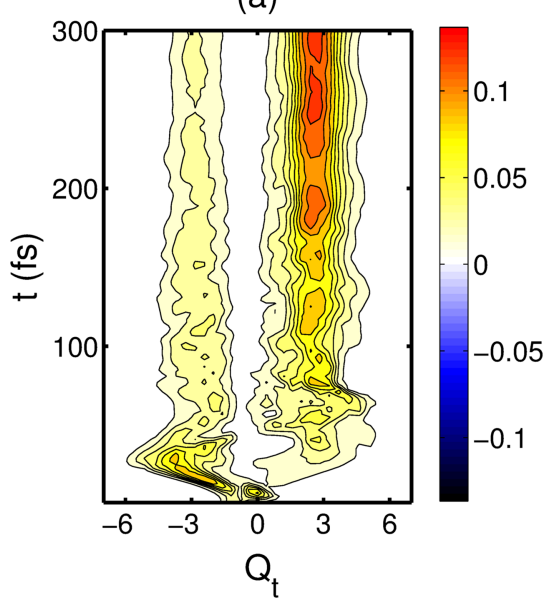

(b)

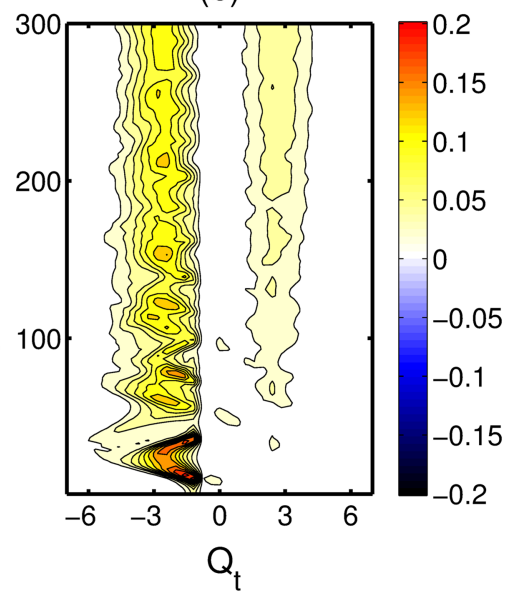

FIG. 4. Time evolution of the probability density $P_{1}^{a d}\left(Q_{t}, t\right)$ of the wave packet on the $|\tilde{e} 1\rangle$ surface for (a) $\Lambda=800 \mathrm{~cm}^{-1}$ and (b) $\Lambda=200 \mathrm{~cm}^{-1}$.

\section{CONCLUSIONS}

In this paper, we have studied the impact of vibrational damping on the dynamics of the electronic wave packet in the vicinity of a CI and its transfer between the potential energy surfaces. The projection of the dynamics onto the tuning and the coupling mode allows us to directly track the nonadiabatic dynamics of the wave packet on the PESs. By this, the direct visualization of the quantum transfer efficiency or the quantum yield and the associated transfer times becomes possible. The quantum yield is evaluated for different values of the vibrational damping strength of the tuning and the coupling modes. It shows that the quantum yield can be significantly enhanced by an increased vibrational coherence of the wave packet. It can reach more than $93 \%$ in the coherent or underdamped case and is reduced to $50 \%$ for the case of an incoherent wave packet. In addition, we find signatures of the geometric (or Berry) phase in the form of a destructive interference in the time-dependent probability density for the case of stronger damping. Moreover, in the case of stronger damping, we observe that the electronic wave packet directly penetrates the higher-lying PES to the lower electronic state $|\tilde{e} 1\rangle$ via tunneling before the remaining parts of the wave packet can pass directly through the CI. Our work helps to clarify the details of the wave-packet dynamics at the seam of the CI, and it provides new approaches for coherently controlling the wave packet traversal in the vicinity of the CI by tuning the vibrational coherence.

\section{ACKNOWLEDGMENTS}

This work was supported by the Max Planck Society and the Excellence Cluster "The Hamburg Center for Ultrafast Imaging-Structure, Dynamics and Control of Matter at the Atomic Scale" of the Deutsche Forschungsgemeinschaft. D.-L. Qi thanks the Fund of ECNU for Overseas and Domestic Academic Visits for support. H.G.D. acknowledges financial support by the Joachim-Hertz-Stiftung Hamburg within a PIER fellowship. H.-G. Duan acknowledges help from Lipeng Chen for the wave packet projection calculations.
${ }^{1}$ W. Domcke, D. R. Yarkony, and H. Köppel, Conical Intersection: Electronic Structure, Dynamics and Spectroscopy (World Scientific, Singapore, 2004). ${ }^{2}$ W. Domcke, D. R. Yarkony, and H. Köppel, Conical Intersection: Theory, Computation and Experiment (World Scientific, Singapore, 2011).

${ }^{3}$ P. Hamm and G. Stock, Phys. Rev. Lett. 109, 173201 (2012).

${ }^{4}$ W. Domcke and D. R. Yarkony, Annu. Rev. Phys. Chem. 63, 325 (2012).

${ }^{5}$ M. Kowalewski, B. P. Fingerhut, K. E. Dorfman, K. Bennett, and S. Mukamel, "Simulating coherent multidimensional spectroscopy of nonadiabatic molecular processes; from the infrared to the x-ray regime," Chem. Rev. (to be published).

${ }^{6}$ D. P. Hoffman and R. A. Mathies, Acc. Chem. Res. 49, 616 (2016).

${ }^{7}$ B. K. Agarwalla, H. Ando, K. E. Dorfman, and S. Mukamel, J. Chem. Phys. 142, 024115 (2015).

${ }^{8}$ P. Kukura, D. W. McCamant, S. Yoon, D. B. Wandschnedier, and R. A. Mathies, Science 310, 1006 (2005).

${ }^{9}$ S. Takeuchi, S. Ruhman, T. Tsuneda, M. Chiba, T. Taketsugu, and T. Tahara, Science 322, 1073 (2008).

${ }^{10}$ C. Fang, R. R. Frontiera, R. Tran, and R. A. Mathies, Nature 462, 200 (2009).

${ }^{11}$ H. Ando, B. P. Fingerhut, K. E. Dorfman, J. D. Biggs, and S. Mukamel, J. Am. Chem. Soc. 136, 14801 (2014).

${ }^{12}$ D. P. Hoffman, S. R. Ellis, and R. A. Mathies, J. Phys. Chem. A 118, 4955 (2014).

${ }^{13}$ D. Polli, P. Altoé, O. Weingart, K. M. Spillane, C. Manzoni, D. Brida, G. Tomasello, G. Orlandi, P. Kukura, R. A. Mathies, M. Garavelli, and G. Cerullo, Nature 467, 440 (2010).

${ }^{14}$ C. Schnedermann, M. Liebel, and P. Kukura, J. Am. Chem. Soc. 137, 2886 (2015).

${ }^{15}$ M. Liebel, C. Schnedermann, G. Bassolino, G. Taylor, A. Watts, and P. Kukura, Phys. Rev. Lett. 112, 238301 (2014).

${ }^{16}$ P. J. M. Johnson, A. Halpin, T. Morizumi, V. I. Prokhorenko, O. P. Ernst, and R. J. D. Miller, Nat. Chem. 7, 980 (2015).

${ }^{17}$ P. J. M. Johnson, M. H. Farag, A. Halpin, T. Morizumi, V. I. Prokhorenko, J. Knoester, T. L. C. Jansen, O. P. Ernst, and R. J. D. Miller, J. Phys. Chem. B 121, 4040 (2017).

${ }^{18}$ V. I. Prokhorenko, A. M. Nagy, S. A. Waschuk, L. S. Brown, R. R. Birge, and R. J. D. Miller, Science 313, 1257 (2006).

${ }^{19}$ M. Liebel and P. Kukura, Nat. Chem. 9, 45 (2017).

${ }^{20}$ W. Domcke and G. Stock, Adv. Chem. Phys. 100, 1 (1997).

${ }^{21}$ S. Hahn and G. Stock, J. Phys. Chem. B 104, 1146 (2000).

${ }^{22}$ A. Kühl and W. Domcke, J. Chem. Phys. 116, 263 (2002).

${ }^{23}$ L. Chen, M. F. Gelin, V. Y. Chernyak, W. Domcke, and Y. Zhao, Faraday Discuss. 194, 61 (2016).

${ }^{24}$ H.-G. Duan and M. Thorwart, J. Phys. Chem. Lett. 7, 382 (2016).

${ }^{25}$ J. Krčmář, M. F. Gelin, D. Egorova, and W. Domcke, J. Phys. B 47, 124019 (2014).

${ }^{26}$ J. Krčmáŕ, M. F. Gelin, and W. Domcke, J. Chem. Phys. 143, 074308 (2015).

${ }^{27}$ M. Sala and D. Egorova, Chem. Phys. 481, 206 (2016).

${ }^{28}$ M. H. Farag, T. C. Jansen, and J. Knoester, J. Phys. Chem. Lett. 7, 3328 (2016).

${ }^{29}$ M. Kowalewski, K. Bennett, K. E. Dorman, and S. Mukamel, Phys. Rev. Lett. 115, 193003 (2015).

${ }^{30}$ D. Keefer, S. Thallmair, S. Matsika, and R. de Vivie-Riedle, J. Am. Chem. Soc. 139, 5061 (2017). 
${ }^{31}$ C. Liekhus-Schmaltz, G. A. McCracken, A. Kaldun, J. P. Cryan, and P. H. Bucksbaum, J. Chem. Phys. 145, 144304 (2016).

${ }^{32}$ M. Richter, F. Bouakline, J. Gonzălez-Vázquez, L. Martínez-Fernández,

I. Corral, S. Patchkovskii, F. Morales, M. Ivanov, F. Martín, and O. Smirnova, New J. Phys. 17, 113023 (2015).

${ }^{33}$ H.-G. Duan, R. J. D. Miller, and M. Thorwart, J. Phys. Chem. Lett. 7, 3491 (2016).

${ }^{34}$ U. Manthe and H. Köppel, J. Chem. Phys. 93, 1658 (1990).

${ }^{35}$ C. Schnedermann, V. Muders, D. Ehrenberg, R. Schlesinger, P. Kukura, and J. Heberle, J. Am. Chem. Soc. 138, 4757 (2016).
${ }^{36}$ C. Meier and D. J. Tannor, J. Chem. Phys. 111, 3365 (1999).

${ }^{37}$ U. Kleinekathöfer, J. Chem. Phys. 121, 2505 (2004).

${ }^{38}$ The wave-packet dynamics has been calculated upto 2.5 ps to quantify the transfer time in the weak damping case.

${ }^{39}$ M. V. Berry, Proc. R. Soc. A 392, 45 (1984).

${ }^{40}$ I. G. Ryabinkin and A. F. Izmaylov, Phys. Rev. Lett. 111, 220406 (2013)

${ }^{41}$ L. Joubert-Doriol, I. G. Ryabinkin, and A. F. Izmaylov, J. Chem. Phys. 139, 234103 (2013).

${ }^{42}$ A. Kelly, and R. Kapral, J. Chem. Phys. 133, 084502 (2010). 